MATEC Web of Conferences 32, 06003 (2015)

DOI: $10.1051 /$ matecconf $/ 20153206003$

C) Owned by the authors, published by EDP Sciences, 2015

\title{
Platform-based Shear Force Sensor
}

\author{
Wei-Chih Wang ${ }^{1,2,4}$, Tien-Hao Chang $^{3}$, Chi-Leung Tsui ${ }^{1}$ \\ ${ }^{1}$ Department of Mechanical Engineering, University of Washington, Seattle, Washington \\ ${ }^{2}$ Department of Electrical Engineering, University of Washington, Seattle, Washington \\ ${ }^{3}$ Department of Biomedical Engineering, National Cheng Kung University, Tainan, Taiwan \\ ${ }^{4}$ Medical Device Innovation Centre, National Cheng Kung University, Tainan, Taiwan
}

\begin{abstract}
In this paper, we will present the development of a flexible fiber optic bend loss sensor for the measurement of plantar pressure and shear stress for diabetic patients. The sensor will allow the measurement of shear stress on the foot, which is a critical parameter in studying diabetic foot ulcers. The basic configuration of the optical sensor systems incorporates a mesh that is comprised of two sets of parallel optical waveguide planes; the planes are configured so the parallel rows of waveguides of the top and bottom planes are perpendicular to each other. The planes are sandwiched together creating one sensing sheet. Two-dimensional information is determined by measuring the loss of light from each of the waveguide to map the overall pressure distribution. The shifting of the layers relative to each other produces different patterns of the sensor output, and shear force information is characterized through repeated training of the sensor and analysis of the training data. The latest development and improvement in the sensors design is presented. Fabrication and sensor characterization results will be presented.
\end{abstract}

\section{Background}

Shear force is an important parameter to evaluate how ulcer is developed for diabetic patient. Most commonly reported techniques utilize electromagnetic or piezoelectric means to deduce this parameter. Previously, attempts have been made using capacitive sensors $[1,2,8,9]$, which measure the horizontal displacement based on the relative capacitance change due to the change in the area of overlap between the top and bottom electrodes. To improve the shear compliance and flexibility, all three reported methods used polydimethylsiloxane (PDMS) as mechanical support and substrate. The advantages of these techniques are they are relatively easy to understand and implement. However, these sensors made of polymers are lack in a robust manufacturing process, as they are currently manually assembled, which makes them difficult to be mass-produced. They also lacked efficient multi-channel capacitance measuring circuits. For a $0.1 \times 1 \times 1 \mathrm{~mm} 3$ sensor, the electronics will require a $0.1 \mathrm{pF}$ or better in sensitivity. It is extremely difficult to achieve this resolution without using an IC based electronics such as capacitor-todigital converter and currently there isn't one available for more 2 channels. Using these techniques also require a very fast DAQ card and smart multiplexing techniques. For the resistive and piezoelectric based shear sensors, these sensors suffer from the same problems as the capacitive sensors in lack of proper electronics support. It also suffers from thermal drift, electrical and mechanical hysteresis, and electromagnetic interference problems that plague most electromagnetic sensors, which we have discussed in our previous papers [3, 4, 5]. To prevent electromagnetic interference caused by the surroundings and also by the human body, Missinne [10], has reported an optical tactile sensors similar to the current capacitive sensors design, where shear and normal displacement is observed by the relative change in the displacement between the top and bottom sensing layer. Herein, displacement in $\mathrm{x}, \mathrm{y}$ and $\mathrm{z}$ directions are detected by an optical pickup made of vertical-cavity surfaceemitting lasers (VCSEL) as the light source and photodiodes as the detectors. This design however still required a large number of output channels for distributive sensing.

Previously, we have reported a distributive pressure/shear sensor using bend loss sensor [3, 4, 5, $6,7,11,12]$. The sensor is composed of two optic fiber meshes which are embedded into a PDMS pad so that fibers' radius of curvatures change when the sensor is pressed. The measurement of light intensity change through bending each fiber provides information about the fiber's changes in curvature. Shear is measured based on the relative position change in the pressure point between the top and bottom layers of the sensor. This sensor has several advantages. One is that it can provide the same number of discrete pressure points compared to commercially available capacitive or resistive based pressure sensors while this optical sensor requires only 2 times the square root of the total number of sensors as the capacitive or resistive sensors. This is due to the fact that each pressure point is defined by an intersection of a row and a column optical waveguide. Therefore the number of output channels is reduced to the total number of waveguides used for the sensor. This unique feature reduces the data acquisition time for each output channel.

In our previous work [13], a 30 channels $(20 \mathrm{x}$ $28 \mathrm{~cm}^{2}$ ) platform based sensor is presented, and 
basic measurement of the shear forces on the foot heel of several different conditions are successfully recorded. To better characterize the sensor data, additional data is collected on different types of foot at different tilting and rotation angles. These data are used as training data and then they are analyzed using Machine Learning techniques, which is used to increase the robustness of the system and ultimately allows the shear sensor to recognize the correct foot shape and the walking direction.

In the report, we will create an algorithm that allows us to predict missing values or outlier values that are produced in the cases of sensor error and fiber fractures. This allows the sensor to have improved fault tolerance.

\section{Methods}

\subsection{Support Vector Machine (SVM)}

Support Vector machine (SVM) is a widely used machine learning tool for various problems [ref]. SVM uses the so-called "maximum margin hyperplane" to overcome over-fitting issue. In the process of finding the maximum margin hyperplane, SVM minimizes both empirical risk and confidence interval. Training samples that are critical to the maximum margin hyperplane are called support vectors. Based on the concepts of maximum margin hyperplane and support vector, SVM has two variants: support vector machine for classification (SVC) and that for regression (SVR). SVR first maps samples into a higher dimensional vector space than the original vector space of the samples with a kernel function. Maximum margin hyperplane is then found in the transformed vector space with the quadratic programming.

Support that $\left\{\left(\mathbf{x}_{1}, y_{1}\right),\left(\mathbf{x}_{2}, y_{2}\right), \ldots\left(\mathbf{x}_{n}, y_{n}\right)\right\}$ is $n$ training samples drawn from the target function $f(\mathbf{x})$ to regress. SVM could be represented as follows.

$$
\min _{\mathbf{w}, b, \xi, \xi^{*}} \frac{1}{2} \mathbf{w}^{T} \mathbf{w}+c \sum_{i=1}^{n} \xi_{i}+c \sum_{i=1}^{n} \xi_{i}^{*}
$$

subjected to

$$
\mathbf{w}^{T} \varphi\left(x_{i}\right)+b-y_{i} \leq \varepsilon+\xi_{i} \text { and } y_{i}-\mathbf{w}^{T} \varphi\left(x_{i}\right)-b \leq \varepsilon+\xi_{i}^{*}
$$

where $\mathbf{w}$ is the weight vector to be figured out; $c$ is a parameter of SVR to control the trade off between the margin error $\left(\xi_{i}\right)$ and the prediction error $\left(\xi_{i}^{*}\right)$; $\varphi(\mathbf{x})$ is the kernel function; and $\varepsilon$ is another parameter of SVR to control the smoothness of the output function. The corresponding dual quadratic programming problem of solving $\mathbf{w}$ is as follows.

$\min _{a, a^{*}} \frac{1}{2}\left(\boldsymbol{\alpha}-\boldsymbol{\alpha}^{*}\right)^{T}\left(\boldsymbol{\alpha}-\boldsymbol{\alpha}^{*}\right)+\varepsilon \sum_{i=1}^{n}\left(\alpha_{i}+\alpha_{i}^{*}\right)+\sum_{i=1}^{n} y_{i}\left(\alpha_{i}+\alpha_{i}^{*}\right)$

subjected to

$\sum_{i=1}^{n}\left(\alpha_{i}-\alpha_{i}^{*}\right)=0$

$0 \leq \alpha_{i}, \alpha_{i}^{*} \leq c$ for $i=1,2, \ldots, n$

Training samples with the corresponding $\alpha_{i}$ larger than zero become support vectors.

\subsection{Relaxed Variable Kernel Density Estimation (RVKDE)}

Relaxed Variable Kernel Density Estimation (RVKDE) is an accurate and efficient kernel density estimation algorithm. By re-constructing the kernel density function of training samples, RVKDE can be used for both classification and regression [ref]. Let $\left\{\mathbf{x}_{1}, \mathbf{x}_{2}, \ldots \mathbf{x}_{n}\right\}$ be a set of sampling instances randomly and independently taken from the distribution governed by $f(\mathbf{x})$ in a $m$-dimensional vector space. Then, with the RVKDE algorithm, the value of $f(\mathbf{x})$ at point $\mathbf{v}$ is estimated as follows.

$\hat{f}(\mathbf{v})=\frac{1}{n} \sum_{\mathbf{x}_{i}}\left(\frac{1}{\sqrt{2 \pi} \cdot \sigma_{i}}\right)^{\alpha} \exp \left(-\frac{\left\|\mathbf{v}-\mathbf{x}_{i}\right\|^{2}}{2 \sigma_{i}^{2}}\right)$,

where

1. $\sigma_{i}=\beta \frac{R\left(\mathbf{x}_{i}\right) \sqrt{\pi}}{\sqrt[m]{(k+1) \Gamma\left(\frac{m}{2}+1\right)}} ;$

2. $R\left(\mathbf{x}_{i}\right)$ is the maximum distance between $\mathbf{x}_{i}$ and its $k s$ nearest training instances;

3. $\Gamma(\cdot)$ is the Gamma function;

4. $\alpha$ by default is the dimension of samples, i.e. $m$, in VKDE (variable kernel density estimation) but relaxed to a parameter in RVKDE;

5. and $\beta$ and $k s$ are parameters to be set through cross-validation.

For classification, a kernel density estimator is constructed for each class to approximate the distribution of samples different classes. Then, a query instance located at $\mathbf{v}$ is predicted to the class that gives the maximum value among the likelihood functions defined as follows: 


$$
L_{j}(\mathbf{v})=\frac{\left|S_{j}\right| \cdot \hat{f}_{j}(\mathbf{v})}{\sum_{h}\left|S_{h}\right| \cdot \hat{f}_{h}(\mathbf{v})}
$$

where $\left|S_{j}\right|$ is the number of class- $j$ training instances, and $\hat{f}_{j}(\mathbf{v})$ is the kernel density estimator corresponding to class- $j$ training instances. For regression, the equation of $\hat{f}(\mathbf{x})$ is slightly adjusted to introduce the function values of each training instance, $f\left(\mathbf{x}_{i}\right)$, denoted as $y_{i}$.

$$
\hat{f}(\mathbf{v})=\sum_{\mathbf{x}_{i}}\left(\frac{1}{\sqrt{2 \pi} \cdot \sigma_{i}}\right)^{\alpha} \cdot y_{i} \cdot \exp \left(-\frac{\left\|\mathbf{v}-\mathbf{x}_{i}\right\|^{2}}{2 \sigma_{i}^{2}}\right)
$$

\subsection{Comparison between SVM and RVKDE}

This subsection reveals some characteristic differences between RVKDE and SVM with the radial basis function (RBF) kernel, which is the most frequently used kernel function in SVM. Both machine learning tools belong to the radial basis function network (RBFN), a special type of neural networks with several distinctive features. The decision function of SVM can be extremely simplified as follows.

$$
f_{\mathrm{SVM}}(\mathbf{v})=\sum_{\mathbf{s}_{i}} y_{i} \cdot \alpha_{i} \cdot \exp \left(-\gamma\left\|\mathbf{v}-\mathbf{s}_{i}\right\|^{2}\right)
$$

where $\mathbf{v}$ is a testing sample; $y_{i}$ is the function value of training sample $\mathbf{s}_{i} ; \alpha_{i}$ is determined by a constrained quadratic optimization; and $\gamma$ is a parameter set through cross-validation. Interestingly, the decision function in equation (8) is very similar to the one in RVKDE using the radial basis function (RBF) kernel:

$$
f_{\mathrm{RVKDE}}(\mathbf{v})=\sum_{\mathbf{s}_{i}} y_{i} \cdot \frac{1}{\sigma_{i}} \cdot \exp \left(-\frac{\left\|\mathbf{v}-\mathbf{s}_{i}\right\|^{2}}{2 \sigma_{i}^{2}}\right),
$$

where $\sigma_{i}$ is the local density of the proximity of $\mathbf{s}_{i}$, estimated by the kernel density estimation algorithm. In equation (9), $\sigma_{i}$ corresponds to $\alpha_{i}^{-1}$ and $\sqrt{\gamma} / 2$ in equation (1). According to equations (1) and (2), the mathematical models of SVM and SVM are analogous. Since $\gamma$ is a parameter in SVM, the main difference between SVM and RVKDE is the criteria to determine $\alpha_{i}$ in Eq. (8) and $\sigma_{i}$ in Eq. (9).

SVM uses support vectors to construct a special kind of linear model, maximum margin hyperplane, that separates the samples of different classes. The $\alpha_{i}$ in SVM is determined based on the global distribution of samples by maximizing the separation between the classes. Conversely, RVKDE uses only $k s$ samples in the proximity of a training instance and thus determines $\sigma_{i}$ based on local information.

\section{Experiment Design}

Tests were conducted on prosthetic feet with three different foot soles as shown in Figure 1, to simulate the different stress distribution created by each foot shape when it is on the sensor.
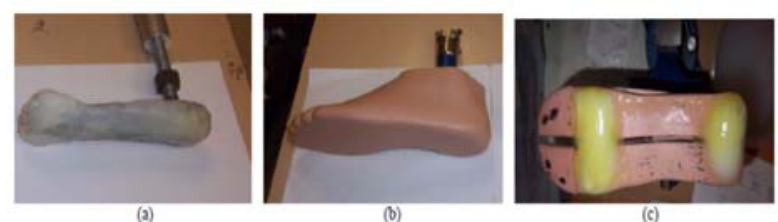

Figure 1. Different kind of full size prosthetic foot (a) Normal healthy foot, (b) Flat foot, (c). Hammer toe foot.

Silicone and Gelatin were used as the main materials for the feet construction except for the flat foot which is a prosthetic foot cover. The full scale foot is then connected to an Interface $1210 \mathrm{AJ}-2$ load cell (2500N max load, .5 $\mathrm{N}$ resolutions) controlled by Instron $5585 \mathrm{H}$ load frame for testing. The applied force is calculated by assuming a person with $100 \mathrm{~kg}$ weight is standing on one foot. Based on the work we done in [13], we are able to obtain the necessary force of $132 \mathrm{~N}$ for special foot, $151 \mathrm{~N}$ for flat foot and $187 \mathrm{~N}$ for normal foot. The yaw angle of the foot and pitch angle of the sensor is adjusted to simulate walking behavior during data collection [13]. The data is collected using Labview program at a sampling rate of $1 \mathrm{kHz}$. The raw data is then going through a use a low pass filter with a cutoff frequency at $100 \mathrm{~Hz}$ to remove high frequency noise. The filtered data is then normalized with respect to its initial DC value to obtain its transmissibility. Ideally, the normalized signal will start from 1 which represents no light loss without any loading on the surface of sensor. The collected data points are then analyzed using the methods in the previous section for characterization. 
There are in total 33769000 "scans" from our shear sensors with different foot shapes, tilting angles and rotation angles at different time since pressing the foot sole. Each scan has 32 normalized signals from the sensor array. To evaluate the accuracy of missing values recovered by machine learning, an experiment was conducted as follows. In the 33769000 scans, 100000 ones were arbitrarily chosen as the training dataset and the remaining 33669000 ones were used as the testing dataset. The training dataset was used to construct a machine learning model. One of the 32 signals of a testing scan was randomly "masked" and then predicted by the machine learning model based on the other unmasked 31 signals. Finally, the difference between the masked and predicted values indicted the prediction error and was used to evaluate the performance of the missing value recovery. Since there is no similar work of missing value recovery in such a shear sensor array, this work introduced a naïve recovery algorithm by predicting any missing signal with the mean of all signals in the training dataset. Four evaluation indices were adopted:

Mean Absolute Error $(\mathrm{MAE})=\frac{1}{n} \sum\left|m_{i}-p_{i}\right|$

Mean Squared Error (MSE) $=\frac{1}{n} \sum\left(m_{i}-p_{i}\right)^{2}$

Relative Absolute Error (RAE) $=\frac{1}{n} \sum \frac{\left|m_{i}-p_{i}\right|}{\left|m_{i}-M\right|}$

and

Relative Squared Error (RSE) $=\frac{1}{n} \sum \frac{\left(m_{i}-p_{i}\right)^{2}}{\left(m_{i}-M\right)^{2}}$

where $n$ is the number of testing samples; $m_{i}$ is the masked signal value of the $i$-the testing sample; $p_{i}$ is the predicted signal value of the $i$-the testing sample; $M$ is the mean of all signals in the training dataset.

\section{Results}

Table 1 shows the evaluation on the errors by Naïve, SVM and RVKDE. On 31 F0 data sets and $31 \mathrm{~F} 10$ datasets, SVM yielded better MAE than RVKDE with by 0.016 and 0.040 (18.2\% and $80.0 \%$ relative to RVKDE's MAE), respectively. SVM also outperformed over RVKDE on $32 \mathrm{R} 0$ data sets, 42 R0D data sets and $35 \mathrm{R} 10$ data sets with $0.017,0.109$ and $0.026 \mathrm{MAE}(12.6 \%, 43.1 \%$ and $18.3 \%$ relative to RVKDE's MAE) advantage.
RVKDE had comparable MAE to SVM on 11 R0TT6 data sets, but SVM still had a slightly advantage of $0.0004 \mathrm{MAE}(0.23 \%$ relative to RVKDE's MAE). Briefly, SVM stably

Table 1. Error Evaluation of Naïve, SVM, and RVKDE

\begin{tabular}{lrrrr}
\hline $\begin{array}{l}\text { Prediction } \\
\text { method }\end{array}$ & MAE & MSE & RAE & RSE \\
\hline Naïve & 0.115 & 0.020 & - & - \\
SVM & 0.093 & 0.011 & 0.813 & 0.563 \\
RVKDE & 0.101 & 0.013 & 0.877 & 0.624 \\
\hline
\end{tabular}

Table 2. Comparison of error between SVM and RVKDE methods

\begin{tabular}{lrrrr}
\hline Type & Count RVKDE & SVM RVKDE - SVM \\
\hline F0 & 31 & 0.090 & 0.074 & 0.016 \\
F0B44 & 2 & 0.114 & 0.131 & -0.017 \\
F10 & 31 & 0.046 & 0.009 & 0.037 \\
R0 & 32 & 0.131 & 0.115 & 0.017 \\
R0D & 42 & 0.253 & 0.144 & 0.109 \\
R0TT6 & 11 & 0.156 & 0.156 & 0.000 \\
R10 & 35 & 0.117 & 0.096 & 0.022 \\
S0 & 32 & 0.079 & 0.026 & 0.053 \\
S0b & 4 & 0.188 & 0.118 & 0.071 \\
S0D & 50 & 0.120 & 0.099 & 0.022 \\
S10 & 31 & 0.102 & 0.050 & 0.052 \\
S2.5D & 49 & 0.060 & 0.123 & -0.063 \\
S5 & 9 & 0.057 & 0.169 & -0.112 \\
S5D & 48 & 0.084 & 0.134 & -0.050 \\
\hline
\end{tabular}

delivered better performance than RVKDE on flat foot $(\mathrm{F})$ and rotation $(\mathrm{R})$ data sets. However, this situation became complicated on shear (S) data sets (shown in Table 2). SVM still yielded better MAE than RVKDE on shear with zero tilting angle data sets (32 S0 data sets, $4 \mathrm{~s} 0 \mathrm{~b}$ datasets and 50 S0D data sets), where SVM had 0.053, 0.071, 0.022 and 0.052 MAE $(66.7 \%, 37.6 \%, 18.2 \%$ and $50.8 \%$ to RVKDE's MAE) advantage. But with more tilting angles, RVKDE outperformed SVM on 49 S2.5D data sets, 9 S5 data sets and 48 S5D data sets with $0.063,0.112$ and $0.050 \mathrm{MAE}(50.9 \%, 66.5 \%$ and $37.5 \%$ relative to SVM's MAE) advantage. However, on the $31 \mathrm{~S} 10$ data sets, SVM retook the advantage by 0.052 MAE ( $50.8 \%$ relative RVKDE's MAE).

As described in the "Comparison between SVM and RVKDE" section, SVM is better in modeling the global trend while RVKDE focuses more on capturing local variations. Therefore, the above 
results suggest that flat foot $(F)$, rotation $(R)$ and shear with zero tilting angle data sets have a common pattern that was learnt by SVM. On shear (S) data sets, increasing tilting angle introduced some local variations that can be captured only by RVKDE.

\section{References}

[1] K. Sundara-Rajan, G.I. Rowe, A.J. Simon, G.K. Klute, W.R. Ledoux, A.V. Mamishev, "Shear sensor for lower

limb prosthetic application,” Proc. BSEC, 1-4 (2009) [2] S. Karki, J. Lekkala, H. Kuokkanen, J. Halttunen, Development of a piezoelectric polymer film sensor for plantar

normal and shear stress measurements, Sensors and Actuators A: Physical, Volume 154, Issue 1, 31 August 2009,

Pages 57-64, ISSN 0924-4247, DOI: 10.1016/j.sna.2009.07.010.

[3] C.Y. Huang, W. Ledoux, W.J. Wu, W.C. Wang, "Composite fiberoptic distributive shear/pressure sensor," IEEE

Sensors Journal, Vol. 7, No. 11, p.1554-1565, 2007. [4] W.C. Wang, W.Y. Hunag, W. Ledoux, P. Reinhall, "Developments of a Microfabricated optical bend loss sensor for

distributive pressure measurement," IEEE Trans. Biomedical Eng., Vol. 55 No.2, p.614-625, 2008.

[5] W.C. Wang, R. Panergo, C.M. Galvanin, W. Ledoux, B Sangeorzan and Per G. Reinhall, "A flexible micromachined optical

sensor for simultaneous measurement of pressure and shear force distribution on foot", Proc. SPIE 5047, 275, 2003.

[6] W.C. Wang, Ledoux W.R., Huang C.Y., Huang C.S., Klute G.K., Reinhall P.G., "Development of a microfabricated

optical bend loss sensor for distributive pressure measurement," IEEE Trans Biomed Eng., 55(2), 614-25, 2008.

[7] W. Soetanto, N. T. Nguyen and W.C. Wang, "Fiber optic plantar pressure/shear sensor", Proc. SPIE 7984, 79840Z,

2011.

[8] J.H. Ho, W.C. Wang, "Development of a polymeric capacitive 3-D tactile sensor," 17 th SPIE NDE health monitoring and Diagnostics, San Diego, CA., 7 - 11 March 2010, SSN10-86

[9] Y.-L. Chou, S.-S. Shi, G.-F. Huang, and T.-S. Lin, "Interface pressure and gait analysis in different walking speeds and on the below-knee amputees with multiple axis foot prosthesis", Biomed. Eng.: Appl. Basis Commun. 15 (5), pp. 38-42, 2003.

[10] J. Missinne, "Flexible miniature shear sensors for prosthetics", SPIE Newsroom. DOI: 10.1117/2.1201104.003664,

27 April 2011,

[11] C.Y. Huang, P. Reinhall, W.J. Wu, W.C. Wang, "A wireless composite optical bend loss sensor for pressure and

shear measurement," SPIE NDE health monitoring and Diagnostics, San Diego, CA., SPIE6177, 2006, [12] C.S. Liu, W.C. Wang, "Composite fiberoptic sensor for shear and plantar pressure measurement," SPIE NDE health

monitoring and Diagnostics, San Diego, CA., SPIE 6532, page 65431k-1 to 10, 2007.

Proc. of SPIE Vol. 8348 83481U-9

[13] Chun-Te Chang ; Chao Shih Liu ; William Soetanto ; Wei-Chih Wang; A platform-based foot pressure/shear sensor. Proc. SPIE 8348, Health Monitoring of Structural and Biological Systems 2012, 83481U (April 26, 2012); doi:10.1117/12.915396. 